\title{
A Negative Effect of Post-Traumatic Growth on Self-perceived Burden of Patients with Lung Cancer During Chemotherapy in China: A Cross-sectional Survey
}

\author{
Liu Guixia ", Zhang Hui, Meng Yun \\ First Affiliated Hospital of Anhui Medical University, Hefei, China \\ Email address: \\ 386901459@qq.com (Liu Guixia),2761145197@qq.com (Zhang Hui),2740032073@qq.com (Meng Yun) \\ ${ }^{*}$ Corresponding author
}

To cite this article:

Liu Guixia, Zhang Hui, Meng Yun. A Negative Effect of Post-Traumatic Growth on Self-perceived Burden of Patients with Lung Cancer During Chemotherapy in China: A Cross-sectional Survey. Psychology and Behavioral Sciences. Vol. 10, No. 1, 2021, pp. 1-9. doi: 10.11648/j.pbs.20211001.11

Received: October 16, 2020; Accepted: October 29, 2020; Published: January 12, 2021

\begin{abstract}
Background: self-perceived burden (SPB) is widespread in cancer patients, which is related to some physical symptoms, but more to psychological ones. Patients with lung cancer have a severe sense of self-burden and post-traumatic growth at different levels. As a protective factor of mental health, post-traumatic growth (PTG) how to influence SPB in cancer patients is rarely reported in the relevant literature. Purpose: To explore the effect of PTG on SPB and its influencing pathway of patients with lung cancer during chemotherapy, and to understand the potential mechanism, the indirect effect of PTG on SPB through illness perception and resilience was also studied. Methods: A total of 345 hospitalized chemotherapy patients with pathological diagnosis of lung cancer were enrolled as subjects. The level of illness perception, resilience, PTG and SPB were measured by the Brief Illness Perception Questionnaire (BIPQ), 10 item Connor Davidson Resilience Scale (CD RISC 10), Posttraumatic Growth Inventory (PTGI) and Self-Perceived Burden Scale for Cancer Patients (SPBS-CP), respectively. And they were analyzed that the effect of PTG on patients' SPB and its influence path. Results: Structural equation modeling results supported the hypothesis mediation model in predicting SPB $\left(\chi^{2}=65.456, \mathrm{df}=34, \chi^{2} / \mathrm{df}=1.925, \mathrm{RMSEA}=0.052\right.$, TLI $=0.980$, $\mathrm{CFI}=0.987)$ with fit indices. It showed that PTG had both direct effect $(\beta=-0.437)$ and indirect effect via illness perception and resilience ( $95 \%$ confidence interval was -1.183 to -0.616 , excluding 0 , total indirect effect was - 0.212) on SPB of lung cancer patients during chemotherapy. Conclusions: PTG plays an obviously negative role in the SPB of patients with lung cancer during chemotherapy, and also has indirect effects on SPB through illness perception and resilience. It is necessary to strengthen multi-disciplinary cooperation and formulate relevant interventions to alleviate SPB by reducing patients' negative illness perception and improving their PTG and resilience.
\end{abstract}

Keywords: Lung Cancer, Self-perceived Burden, Post-traumatic Growth, Illness Perception, Resilience

\section{Introduction}

SPB is a sense of "burden to others", which is defined as care recipients' empathic concern derived from the impact on others of their own illness and care needs, resulting in the feelings of guilt, distress, responsibility and the diminished sense of self [1].

SPB is ubiquitous in cancer patients [2,3]. It is related to some physical symptoms, but more commonly to psychological ones [2]. If the symptoms burden of patient is more severe and the mental health status (e.g., anxiety, depression) more worse, the SPB is heavier [4-7]; on the contrary, the SPB is lighter if patients' quality of life is good and coping styles are positive [8]. Other studies also have shown that the SPB is more serious if the patient depended on the older caregivers or palliative treatment to maintain their lives, knew more about disease-related knowledge or perceived more equity-inequity $[5,9,10]$.

SPB increases the physical, psychological risk and survival problems of patients, and affects the prognosis of cancer [3]. SPB can also have a significant impact on patients' decisions making, inducing them to choose not to start treatment, refuse 
medical means to prolong life, have a reduced desire for survival, desire to accelerate the process of death, and/or prefer to receive care in the medical institutions rather than at home at the end of life [11].

Cancer is a stressful and traumatic event that can bring about an adverse impact on psychological well-being. It may also catalyze a range of positive responses, including personal, interpersonal and spiritual improvements, commonly known as post-traumatic growth (PTG) [12]. PTG refers to the positive psychological changes and growth exceeding the "baseline" level of pre-traumatic psychological functioning, which appears following a serious, unbearable, or traumatic event [13, 14]. It is determined by changed subjective cognition, such as altered priorities, perceived new possibilities, greater life appreciation, changed spirituality, enhanced interpersonal relationships and personal strength [15]. Therefore, individuals with high PTG level have enough ability to deal with their life problems, and can improve their mental health level by focusing on positive outcomes of trauma and improving coping strategies, so that they can easily adapt to the process of cancer [16].

At present, SPB studies less in oncology except for palliative care [11], PTG research covers natural disasters, sexual assault, AIDS, cardiovascular diseases, cancer and so on [17]. PTG was commonly seen in lung cancer patients [18], and $97.98 \%$ of them had SPB of different degrees [19]. Chemotherapy, as an important treatment for lung cancer patients, requires perfect physical and mental cooperation of patients in order to play the best effect. As a protective factor of mental health, PTG how to influence SPB in cancer patients is rarely reported in the relevant literature. Equity theory and the psychology of loss maybe provide a useful model for this discussion. Equity theory hypothesizes the balance between benefits and contributions of individuals. When individuals perceive over benefits or less contributions, it occurs the feelings of guilt or worry about becoming a burden to their caregiver [20]. In other words, SPB may occur when individuals loss something (e.g., health, opportunities or abilities to give to others). Psychology of loss, an area within positive psychology, emphasizes the study of the resources used by individuals adapting to loss and the ways turned losses into personal growth and strength [21], which consistent with the concept and construct of PTG. Therefore, PTG may make up for this loss, that is to say, higher levels of PTG maybe reduce patients' perception of SPB. The purpose of this study is to explore whether this hypothesis is true and to what extent PTG affects SPB in patients with lung cancer during chemotherapy.

Studies have shown that PTG was negatively associated with illness perception and positively associated with resilience $[22,23]$. Consequently, as important psychological variables, this study also examined illness perception and resilience at the same time.

Based on what has been discussed above, we hypothesized that PTG would be associated with SPB. Illness perception and resilience were hypothesized to mediate between PTG and
SPB, such that PTG would be associated with illness perception and resilience, which would then be associated with SPB.

\section{Methods}

\subsection{Sample Size}

The SPB level of lung cancer patients is high. Combining with literature and sample size formula $\mathrm{N}=\mathrm{Z}^{2} \times(\mathrm{P} \times(1-\mathrm{P})) / \mathrm{E}^{2}$, the calculated sample size of this study is at least 106 cases when the probability value is $50 \%$, the allowable error value is $10 \%$, and the missing rate is $10 \% \sim 15 \%$. Among them, $\mathrm{N}$ is the sample size, $\mathrm{Z}$ is the statistic, $\mathrm{E}$ is the error value, $\mathrm{P}$ is the probability value, and $Z$ is equal to 1.96 when the confidence is $95 \%$. In order to obtain stable results in structural equation model analysis, the best number of samples to be tested is more than 200 [24].

\subsection{Participants}

In this study, the cross-sectional survey was conducted by convenience sampling method. The subjects were lung cancer patients who received chemotherapy from February 2018 to December 2018 at a grade iii-a hospital in Anhui Province. During the period of hospitalization, patients received quality-overall responsibility nursing. Patients inclusion criteria: The patients were clear-minded, aged over 18 years old, and pathologically diagnosed as lung cancer. Patients exclusion criteria: All patients were excluded when they had language communication disorder, lung cancer with psychiatric symptoms or had a history of psychosis. A total of 360 questionnaires were distributed in this study, and 15 invalid ones were excluded (owing to the similarity and regularity answers to different items in the questionnaire). The final effective sample size was 345, with an effective questionnaire rate of $95.83 \%$. The research program was approved by the ethics committee of the hospital, and all the subjects or their families signed the informed consent.

\subsection{Instruments}

\subsubsection{Brief Illness Perception Questionnaire (BIPQ)}

Brief Illness Perception Questionnaire of 8 items is mainly used to evaluate patients' perception and cognition of disease. Each item is scored from 0 (no impact) to 10 (serious impact) points (reverse scoring of items 3, 4 and 7), with a total score of 0 to 80 . The higher the score, the more negative perception the patients have. The Cronbach' $\alpha$ coefficient of the questionnaire is 0.77 [25], which is 0.76 in the current study.

\subsubsection{0- item Connor Davidson Resilience Scale (CD-RISC 10)}

This scale is used to assess the resilience level of individuals in stress response. There are 10 items which have a score of 0 (never) $\sim 4$ (always) and a total score of $0 \sim 40$. The higher the score, the better the resilience the patients have. It's Cronbach' $\alpha$ coefficient is 0.87 [26], and 0.81 in the current study. 


\subsubsection{Posttraumatic Growth Inventory (PTGI)}

The Posttraumatic Growth Inventory gets 20 items, including five dimensions: life perception, new possibilities, personal strength, relationship with others, and self-transformation. It is a measure of the level of positive change after an individual's trauma. Each item counts 0 (not at all) to 5 (very much) points, with a total score of 0 to 100 points. All entries are scored in the right direction. High scores indicate more growth after trauma. The Cronbach's a coefficient of PTGI and each dimension is 0.61 $\sim 0.87$ [27], while it is $0.65 \sim 0.82$ in this study.

\subsubsection{Self-Perceived Burden Scale for Cancer Patients (SPBS-CP)}

The scale is mainly used to measure the SPB level of cancer patients. There are 21 items which consist of four dimensions: economic/family burden, psychological/emotional burden, care burden and treatment burden. Each item was scored from 1 (never) to 5 (always) points, with a total score of $21 \sim 105$. A higher score means a higher level of patient's SPB. According to the total score of SPBS-CP, the burden is divided into four categories: 1) no significant SPB: the total score is less than 30; 2) mild SPB: the total score is $30 \sim 49 ; 3$ ) moderate SPB: the total score is $50 \sim 69 ; 4)$ severe SPB: the total score is 70 or more. The Cronbach's a coefficient of SPBS-CP and each dimension is $0.79 \sim 0.94$ [28], which is $0.70 \sim 0.86$ in the current study.

\subsection{Investigation Methods}

Cross-sectional survey was used in this study. All the scales were issued on the day the patient was dismissed from the hospital. The patients filled in the questionnaires themselves after the trained researchers utilized the unified guidance language to explain the purpose of the study, the method of filling out, and the precautions. For those who were inconvenient to fill in the entry, the investigator read the items one by one and asked the patient to choose and the investigator recorded it on his own behalf. The scale was retrieved by the researcher on the spot and checked for validity.

\subsection{Statistical Analysis Methods}

The descriptive statistic, single factor analysis and Pearson correlation analysis were carried out in SPSS 17.0 software for major variables. $P$-values $<0.05$ (double sided) were considered to have statistical significance. The missing values (only $0.01 \sim 0.04 \%$ ) were analyzed by expectation maximization (EM) algorithm in SPSS.

Structural equation model (SEM) was constructed with Amos 17.0 to evaluate the applicability of the hypothetical model in explaining the correlations among illness perception, resilience, post-traumatic growth and self-perceived burden. An initial hypothesis model was established through reference to related literature $[3-7,10,13]$ and the results of single factor analysis, which was used the payment method, cancer stage and PTG as independent variables, SPB as dependent variables, BIPQ and CD-RISC10 as intermediary variables. Then, the parameters of the hypothetical model were estimated with the maximum likelihood method. According to the model correction index, the path with no significant parameter $(P>0.05)$ is deleted and the model was revised repeatedly.

Four model fitting indices were reported to evaluate the hypothetical model, which include the chi-square to degrees of freedom $\left(\chi^{2} / \mathrm{df}\right)<3$, the comparative fit index $(\mathrm{CFI}) \geq 0.90$, the root mean square error of approximation (RMSEA) $\leq 0.08$, Tucker-Lewis index (TLI) $>0.90[24]$.

In order to guarantee the stability of path Coefficient estimation, 2000 bootstrap replications of the mediation model are also carried out. If the $95 \%$ of bias-corrected confidence intervals did not contain zero, the indirect effect indicated statistically significant.

\section{Results}

\subsection{Demographic Characteristics}

The average age of lung cancer patients during chemotherapy period was $(62.09 \pm 9.29)$ years old, and the time of diagnosis was $3(1,5)$ months. $72.2 \%$ of the patients were males, $91.9 \%$ were married, and most of them were elementary school and below educated. Table 1 presents the demographic characteristics of patients.

Table 1. Characteristics of lung cancer patients during chemotherapy $(N=345)$.

\begin{tabular}{|c|c|c|c|}
\hline variables & Variable layering & $N$ & $(\%)$ \\
\hline \multirow{4}{*}{ Age (years) } & $\leq 44$ & 5 & $(1.4)$ \\
\hline & $45 \sim 59$ & 115 & $(33.3)$ \\
\hline & $60 \sim 74$ & 196 & $(56.8)$ \\
\hline & $\geq 75$ & 29 & $(8.4)$ \\
\hline \multirow{2}{*}{ Gender } & Male & 249 & $(72.2)$ \\
\hline & Female & 96 & $(27.8)$ \\
\hline \multirow{3}{*}{ Marital status } & Married & 317 & $(91.9)$ \\
\hline & Divorced & 1 & $(0.3)$ \\
\hline & Widowed & 16 & $(4.6)$ \\
\hline \multirow{3}{*}{ Education level } & Elementary school and below & 247 & $(71.6)$ \\
\hline & High school or secondary school & 88 & $(25.5)$ \\
\hline & College or undergraduate & 10 & $(2.9)$ \\
\hline \multirow{3}{*}{ Patients' payment way } & Medical insurance & 193 & $(55.9)$ \\
\hline & New rural cooperative medical care & 108 & $(31.3)$ \\
\hline & Other ways & 44 & $(12.8)$ \\
\hline
\end{tabular}


Liu Guixia et al:: A Negative Effect of Post-Traumatic Growth on Self-perceived Burden of Patients with Lung Cancer During Chemotherapy in China: A Cross-sectional Survey

\begin{tabular}{llll}
\hline variables & Variable layering & N & $(\mathbf{\%})$ \\
\hline & Worker & 34 & $(9.9)$ \\
& Farmer & 176 & $(51.0)$ \\
& Staff/medical/teacher & 16 & $(0.9)$ \\
& Cadre & 3 & $(12.5)$ \\
& Other occupations & 43 & $(38.8)$ \\
& Squamous cell carcinoma & 134 & $(38.8)$ \\
& Adenocarcinoma & 134 & $(19.4)$ \\
Cancer type & Small cell carcinoma & 67 & $(2.9)$ \\
& Other types of cancer & 10 & $(27.8)$ \\
& Stage I II/ limited-stage & 96 & $(21.4)$ \\
Cancer staging & Stage III & 74 & $(50.7)$ \\
& Stage IV / extensive-stage & 175 & $(28.1)$ \\
Number of chemotherapy & the first time & 97 & $(48.7)$ \\
& $2 \sim 4$ times & 168 & $(23.2)$ \\
& 5th or above & 80 & $(27.2)$ \\
Drug emetic properties & High & 94 & $(50.1)$ \\
& Medium & 173 & $(22.6)$ \\
\hline
\end{tabular}

\subsection{Single Factor Analysis of Overall Self-perceived Burden}

Total SPBS-CP score of patients with lung cancer during chemotherapy was $(66.08 \pm 18.83)$, and the scores of economic/family burden, psychological/emotional burden, care burden and treatment burden were $(23.91 \pm 6.63),(16.57 \pm$ $6.60),(14.15 \pm 4.19)$ and $(11.43 \pm 4.54)$, respectively. Among burdens $(20.3 \%), 114$ moderate burdens (33.0\%), and 157 severe burdens $(45.5 \%)$. The total SPBS-CP score was higher than the normal model [28] $(n=330$, the total score is $59.10 \pm$ $18.12, t=6.891, P=0.000$ ), and univariate analysis showed that only the mode of payment and cancer stage affected patients' SPB $(\mathrm{P}<0.05)$. The results of single factor analysis of total SPBS-CP score of patients are shown in Table 2.

Table 2. Single factor analysis of overall self-perceived burden in patients with lung cancer during chemotherapy.

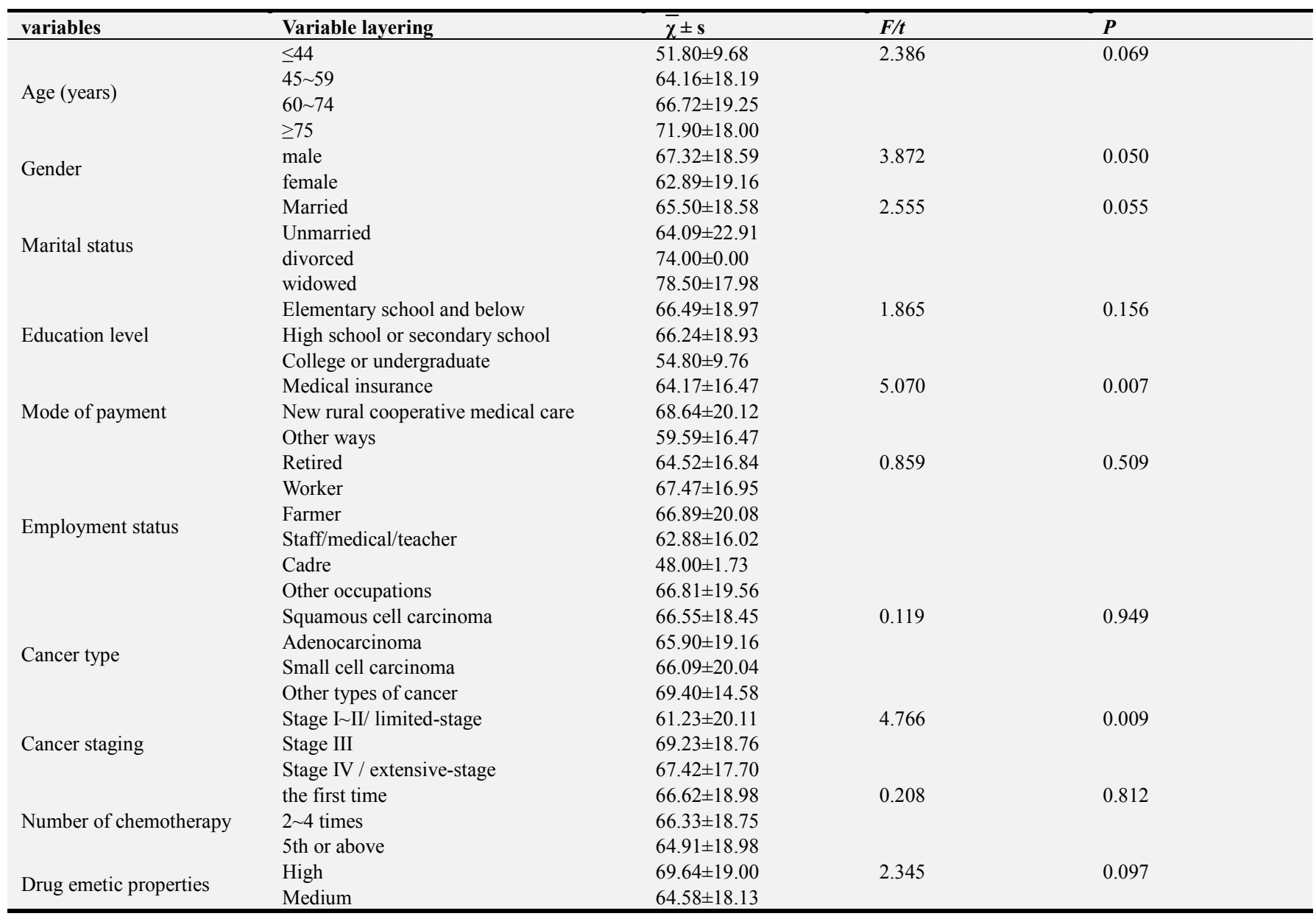




\begin{tabular}{llccc}
\hline variables & Variable layering & $\bar{\chi} \pm \mathbf{s}$ & $\boldsymbol{F} / \boldsymbol{t}$ & $\boldsymbol{P}$ \\
\hline & Low & $65.14 \pm 19.78$ & & \\
\hline
\end{tabular}

\subsection{Correlation Analysis Among SPBS-CP, PTGI, BIPQ and CD-RISC10}

The total score and each dimension of SPBS-CP in lung cancer patients during chemotherapy were negatively related to CD-RISC10, the total score and its dimensions of PTGI $(r=-0.272 \sim-0.558, \quad P<0.01)$, positively related to BIPQ $(r=0.419 \sim 0.553, P<0.01)$, and the specific results are shown in Table 3.

Table 3. Correlation among SPBS-CP, PTGI, BIPQ and CD-RISC10 in lung cancer patients during chemotherapy (r).

\begin{tabular}{llllll}
\hline variables & Score $(\bar{\chi} \pm \mathbf{s})$ & B1 & B2 & B3 & B4 \\
\hline P1 & $17.29 \pm 5.85$ & $-0.404^{* *}$ & $-0.550^{* *}$ & $-0.352^{* *}$ & $-0.537^{* *}$ \\
P2 & $10.04 \pm 4.37$ & $-0.373^{* *}$ & $-0.488^{* *}$ & $-0.311^{* *}$ & $-0.476^{* *}$ \\
P3 & $8.11 \pm 3.11$ & $-0.402^{* *}$ & $-0.535^{* *}$ & $-0.331^{* *}$ & $-0.554^{* *}$ \\
P4 & $8.99 \pm 3.00$ & $-0.342^{* *}$ & $-0.488^{* *}$ & $-0.272^{* *}$ & $-0.476^{* *}$ \\
P5 & $9.19 \pm 3.14$ & $-0.327^{* *}$ & $-0.342^{* *}$ & $-0.300^{* *}$ & $-0.306^{* *}$ \\
P & $53.61 \pm 17.71$ & $-0.428^{* *}$ & $-0.557^{* *}$ & $-0.365^{* *}$ & $-0.547^{* *}$ \\
BIPQ & $46.54 \pm 10.81$ & $0.432^{* *}$ & $0.471^{* *}$ & $0.419^{* *}$ & $-0.376^{* *}$ \\
CD-RISC10 & $21.69 \pm 6.64$ & $-0.418^{* *}$ & $-0.510^{* *}$ & $-0.300^{* *}$ & $0.553^{* *}$ \\
\hline
\end{tabular}

Note: **. Significant correlation was found at the level of 0.01 (bilateral). B1: economic/family burden, B2: Psychological/emotional burden, B3: care burden, B4: treatment burden, B: the total score of SPBS-CP. P1: Life perception, P2: new possibilities, P3: personal strength, P4: relationship with others; P5: self-transformation, P: the total score of PTGI.

\subsection{Pathway Analysis of PTG Influencing SPB}

Based on the preliminary analysis, the payment method and cancer stage were included in the model because it was shown significantly correlated with SPB. However, according to the model modification index, the path parameters of the two variables were not statistically significant $(P>0.05)$, so they were deleted and then the model was modified repeatedly. Finally, a structural equation model with better index fitting was obtained $\left(\chi^{2}=65.456, d f=34, \chi^{2} / d f=1.925\right.$, $\mathrm{RMSEA}=0.052$,
$\mathrm{TLI}=0.980, \quad \mathrm{CFI}=0.987$ ). Bootstrap mediating effect test showed that $95 \%$ confidence interval of mediating effect from PTG to SPB was (- 1.183 - 0.616), and the interval did not contain 0 , so the mediating effect was significant. Mediation effect analysis suggested that PTG not only directly affected SPB, but also indirectly affected it through disease perception and resilience. The specific results are shown in Figure 1 and Table 4.

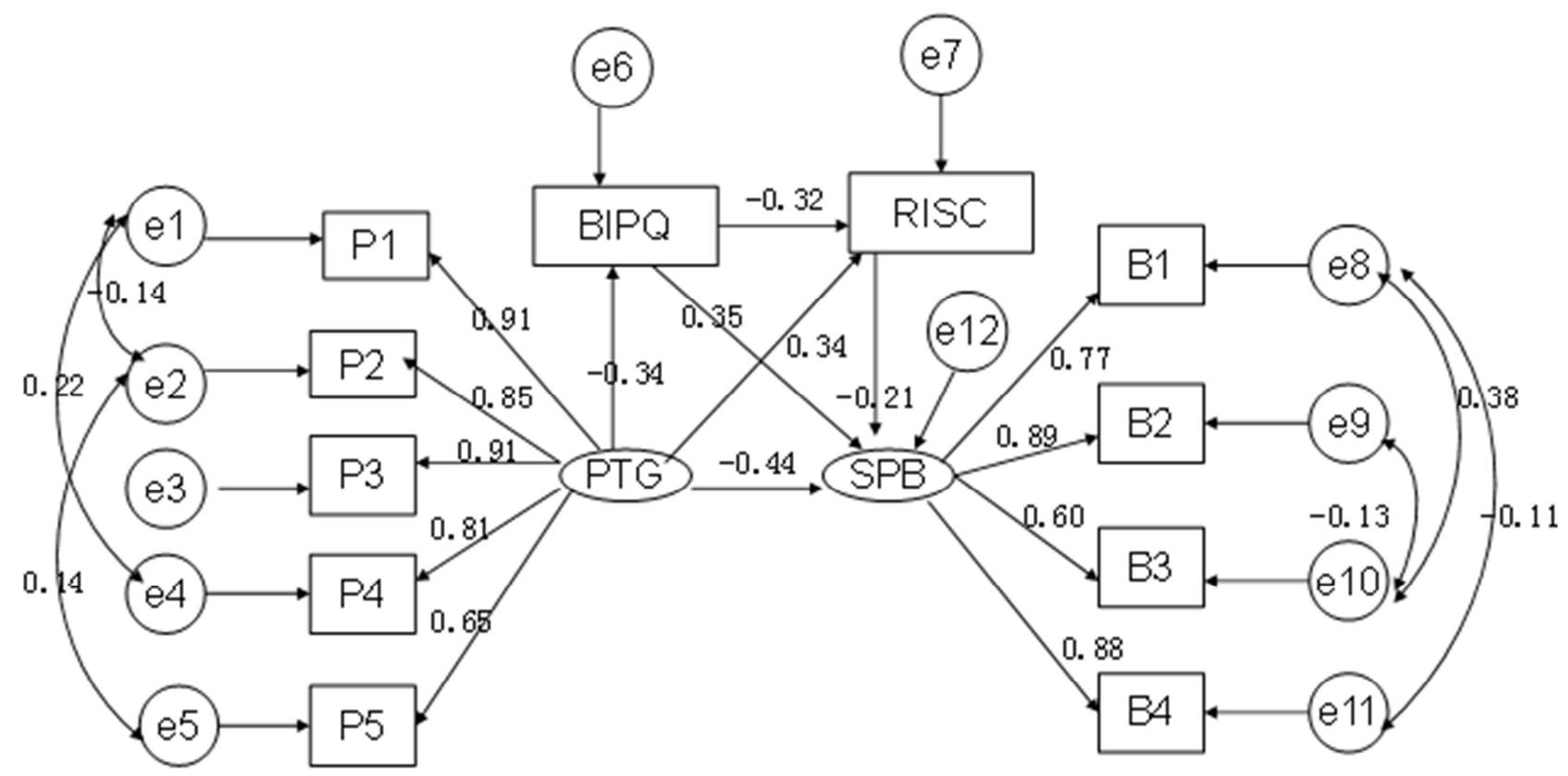

Figure 1. Pathway analysis the effect of PTG on SPB in lung cancer patients during chemotherapy.

Note: B1: economic/family burden, B2: Psychological/emotional burden, B3: care burden, B4: treatment burden; P1: Life perception, P2: new possibilities, P3: personal strength, P4: relationship with others; P5: self-transformation. 
Table 4. Intermediate effect of PTG on SPB in lung cancer patients during chemotherapy.

\begin{tabular}{lll}
\hline Impact Path & Standardized effect values & ratio \\
\hline $\mathrm{PTG} \rightarrow \mathrm{SPB}$ & -0.437 & $(-0.437 /(-0.649)=67.33 \%$ \\
$\mathrm{PTG} \rightarrow \mathrm{CD}-\mathrm{RISC} \rightarrow \mathrm{SPB}$ & $0.35 \times(-0.21)=-0.0735$ & $(-0.0735 /(-0.649)=11.33 \%$ \\
$\mathrm{PTG} \rightarrow \mathrm{BIPQ} \rightarrow \mathrm{SPB}$ & $(-0.34) \times 0.34=-0.1156$ & $(-0.1156 /(-0.649)=17.81 \%$ \\
$\mathrm{PTG} \rightarrow \mathrm{BIPQ} \rightarrow \mathrm{CD}-\mathrm{RISC} \rightarrow \mathrm{SPB}$ & $(-0.34) \times(-0.32) \times(-0.21)=-0.0228$ & $(-0.0228 /(-0.649)=3.51 \%$ \\
$\mathrm{T}$ tal effect of PTG on SPB & $(-0.437)+(-0.0735)+(-0.1156)+(-0.0228)=-0.649$ & $100 \%$ \\
\hline
\end{tabular}

\section{Discussions}

\subsection{Analysis of SPB in Patients with Lung Cancer During Chemotherapy}

The results of this study showed that $98.8 \%$ of lung cancer patients experienced SPB of different degrees in chemotherapy stage, which was in the middle level, but higher than Ren Yanyan's investigations on the SPB of 330 mid-advanced cancer patients [28]. The severity of SPB was heavier than that of amyotrophic lateral sclerosis, hypertension and advanced cancer patients [7, 10, 29]. This indicated that the SPB level of lung cancer patients was high and the severity was serious during chemotherapy, which might be related to their serious negative psychology, symptoms burden and perceived imbalance in interpersonal communication.

Through a qualitative interview study of patients with advanced cancer, McPherson CJ [1] found that participants' experience of SPB was mainly reflected in two related themes: " Concern for Others " and "Implications for Self ". "Concern for Others" involves the specific burden that patients impose on people around them due to their own diseases and their adverse consequences, including physical burdens, social burdens, economic burdens, as well as worries about the future, and the possible impact of their deaths on those around them. "Implications for Self" mainly consists of three self-thoughts and emotions that burden to others: a sense of responsibility for creating difficulties to others, leading to distress and a weakened sense of self.

Lung cancer is the leading cause of death in cancer patient worldwide because of its high morbidity and mortality [30], its early diagnosis rate is less than $20 \%$ [31], and the 5 -year survival rate is only $4 \% \sim 15 \%[32,33]$, which is the lowest among all cancer types [34]. After the diagnosis of cancer, patients often suffer from psychological distress, fear of death, depression, despair and even suicide, as while as the side effects caused by treatment make their health conditions worse more [35]. All these make their physical and mental trauma obvious, and urgently need the support, companionship and care of others, especially their families. They are often prone to create psychological feelings of dragging their families and becoming family burdens. According to equity theory, cancer patients often perceive inequity, resulting in a sense of guilt that gives less but benefits too much [36], which is supported by the results of this study. Research showed that inequity perception was positively correlated with SPB, and the more benefits advanced cancer patients got from care, the more severe the SPB was [5]. Study of stroke survivors also indicated that people who benefited too much from relationships experienced higher SPB levels than those who thought they benefited too little or equal [37]. The results of this study suggested that cancer staging and payment methods affected the SPB of patients, and the SPB was higher in patients with advanced lung cancer and new rural cooperative medical care. In the study, $72.1 \%$ patients were in advanced cancer staging, and the proportion of self-paid medical expenses of the patients in the new rural cooperative medical system was as high as $70 \%$. Study has shown that the average cost per lung cancer patient in China is more than \$9891 [18], 83.7\% of the patients feel financial difficulties [38]. The SPB was more severe when patients had cancer staging later and medical expenses were more [19,39], and patients with out-of-pocket expenses felt more burden of care [10]. This is suggested that clinical medical staff should pay more attention to the patients with advanced lung cancer and those with the high proportion of out-of-pocket expenses, strengthen multi-disciplinary cooperation, and at the same time, actively care about the patients' psychology while alleviating the burden of symptoms, and intervene in time to balance the interpersonal communication of patients so as to reduce their SPB level.

\subsection{Analysis of the Effect of Post-traumatic Growth on Self-perceived Burden in Patients with Lung Cancer During Chemotherapy}

In this study, the PTG score of lung cancer patients in chemotherapy stage was similar to that of breast cancer patients [40], which was at a low level in general. PTG had a certain negative predictive effect on SPB, and the direct effect was obvious.

Medical illness can be regarded as a psychological change. People with severe illness may experience a process of breakdown due to a lack of understanding of the disease and a painful awareness of its severity $[17,22]$. The seriousness of lung cancer itself and its adverse prognosis, together with a series of side effects of chemotherapy, may increase the patients' sense of illness uncertainty, symptom burden and psychological distress. In the struggle against cancer diagnosis and treatment, when the disease is too uncertain and the patient is in a state of lasting pain and on the verge of collapse, it may be difficult for them to appreciate the small things in life, to discover new possibilities, to be more compassionate and willing to build closer relationships with others. Similarly, they are also rarely willing to improve adaptive resources, deepen self-awareness, re-adjust personal values and enhance 
resilience to subsequent stress or trauma. They believe that individual strength is weak, new possibilities are small, life is dim, and no matter how many changes can be made, it is difficult to reverse the outcome of life being about to end. Therefore, it is difficult for patients to gain growth from this specific event, resulting in a serious psychological and emotional burden. They believe that their own existence is the main cause of the serious economic burden on the family and has greatly dragged down it. This reminds that clinical staff should guide the patients with lung cancer in the period of chemotherapy to correctly evaluate the impact of lung cancer chemotherapy on their lives, to actively recognize and reconstruct the value of life. At the same time, clinical staff should also activate patient's social and family support system in time, harmonious interpersonal relationship, and create a good medical and family care atmosphere, so that patients can achieve a higher level of growth in the event of lung cancer chemotherapy.

This study also found that PTG also plays an indirect role in SPB through illness perception and resilience. Illness perception refers to patients' cognitive assessment and personal understanding of disease status and its potential consequences [41]. PTG derives from the coping process after exposure to traumatic events and is determined by changing subjective perception [15]. By changing their cognition and behavior in an effort to find the underlying meaning of traumatic events, individuals can successfully master or control traumatic events and maintain or enhance their self-esteem [12]. The study showed that the negative perception of breast cancer patients was significantly associated with emotional distress and lower physical and mental quality, and higher PTG cushioned negative effects on the quality of psychological and social life $(P<0.01)$, as well as depression $(P<0.06)$, which suggested that positive changes in the cancer experience can protect women from negative cognitive impacts in adapting to breast cancer [42]. Resilience is an ability to successfully cope with negative emotions caused by pressure [13], a series of personality traits or qualities that help achieve adaptive results [43], an important protective factor against psychological distress [22], and the strongest determinant of PTG [44], which is closely related to mental health. A study has shown that there is a positive correlation between resilience and PTG [22]. Lung cancer patients with high PTG level may have stronger resilience, less negative perception, better ability to cope with stress and manage adversity, and a higher sense of self-efficacy, so they may have fewer thoughts of being useless and burdensome to others. Previous study has also suggested that resilience can predict and positively reflect the occurrence and development of SPB [19]. Resilience can be gained through training and intervention. Assessing the level of patients' resilience and adjusting intervention measures to promote the recovery of their resilience can be conducive to improve the effectiveness of intervention measures for patients' psychological symptoms [45]. At the same time, improving health outcomes by strengthening personal and social resources and enhancing the effectiveness of coping strategies may be an effective intervention strategy, which can promote the growth of cancer patients after trauma [22]. If patients can better understand the disease and its impact on themselves, they will show less negative emotions and better cooperate with the treatment [46, 47]. Rational illness perception can promote patients to choose and adhere to a healthy lifestyle and improve their quality of life. Therefore, clinical medical staff should pay attention to the evaluation of resilience of lung cancer patients during chemotherapy and strengthen multidisciplinary cooperation. Those with poor resilience should be educated, trained and counseled in a timely manner so as to maintain their mental health level and reduce the idea that they are burdened to others and their families. In addition, in the daily mental health education of patients, we should actively cultivate their optimistic personality, make them correctly attributable and understand the cancer and cancer chemotherapy events and the impact of such events on themselves, so that they can gain more growth in trauma, reduce negative emotions, actively accept treatment and nursing, thus reducing the feeling of psychological burden.

\section{Conclusions}

In summary, PTG has a negative effect on the SPB of patients with lung cancer undergoing chemotherapy. It is necessary to strengthen multidisciplinary cooperation and formulate relevant interventions to alleviate patients' self-perceived burden by improving their post-traumatic growth, resilience and reducing illness perception. However, the subjects of this study are from the same center and the sample size is limited. At the same time, the disease and psychological changes of patients are dynamic development, so the results of this study have some limitations. In order to verify the validity and scientificity of the above results, we need to carry out large-scale multi-center follow-up studies on patients with different chemotherapy cycles. Meanwhile, we should actively seek feasible and effective interventions (e.g., strengthening health education, developing harmonious medical-patient relationship, enhancing patients' emotional and information support, reducing their negative disease cognition, etc) to reduce patients' burden of self-perception and improve their mental health level and quality of life.

\section{Conflict of Interest}

All the authors do not have any possible conflicts of interest, including financial interests or relationships.

\section{Acknowledgements}

This study was supported by the First Affiliated Hospital of Anhui Medical University, and all data collection was completed in the department of respiratory and critical care medicine of the hospital. Thanks for the strong support from the department director and head nurse, as well as the active cooperation of all patients and their families involved. 


\section{References}

[1] McPherson CJ, Wilson KG, Murray MA (2007). Feeling like a burden: exploring the perspectives of patients at the end of life. Soc Sci Med 64: 417-427.

[2] McPherson CJ, Wilson KG, Lobchuk MM, Brajtman S (2007). Self-perceived burden to others: patient and family caregiver correlates. J Palliat Care 23: 135-142.

[3] Lofaso CR, Weigand DA (2014). Individual Characteristics and Self-Perceived Burden in Cancer Patients. Curr Psychol 33 174-184.

[4] Kowal J, Wilson KG, McWilliams LA, Péloquin K, Duong D (2012). Self-perceived burden in chronic pain: relevance, prevalence, and predictors. Pain 153: 1735-1741.

[5] Miki (2017). Physical and Cognitive Factors Associated with Self-Perceived Burden in Patients with Advanced Cancer. Hiroshima J Med Sci 66: 55-59.

[6] Tang ST, Hsieh CH, Chiang MC, Chen JS, Chang WC, Chou WC, Hou MM (2017). Impact of high self-perceived burden to others with preferences for end-of-life care and its determinants for terminally ill cancer patients: a prospective cohort study. Psychooncology 26: 102-108.

[7] Lin Q, Fu MX, Yang LN, Wu JJ, Wang HY, Zhang XG (2019). Mental health is as important as physical health: The degree and influencing factors of self-perceived burden in elderly patients with essential hypertension. TMR Integrative Nursing 3: $27-34$.

[8] Kuo SC, Chou WC, Hou MM, Wu CE, Shen WC, Wen FH, Tang ST (2018). Changes in and modifiable patient- and family caregiver-related factors associated with cancer patients' high self-perceived burden to others at the end of life: A longitudinal study. Eur J Cancer Care (Engl) 27: e12942.

[9] Lee JE, Shin DW, Cho J, Yang HK, Kim SY, Yoo HS, Jho HJ, Shin JY, Cho B, Park K, Park JH (2015). Caregiver burden, patients' self-perceived burden, and preference for palliative care among cancer patients and caregivers. Psychooncology 24: $1545-1551$.

[10] Geng D, Ou RW, Miao XH, Zhang LH, Wei QQ, Chen XP, Liang Y, Shang HF, Yang R (2016). Patients' self-perceived burden, caregivers' burden, and quality of life for ALS patients: a cross-sectional study. J Clin Nurs 26: 3188-3199.

[11] Libert Y, Borghgraef C, Beguin Y, Delvaux N, Devos M, Doyen C, Dubruille S, Etienne AM, Liénard A, Merckaert I, Reynaert C, Slachmuylder JL, Straetmans N, Van Den Neste E, Bron D, Razavi D (2017). Factors associated with self-perceived burden to the primary caregiver in older patients with hematologic malignancies: an exploratory study. Psychooncology 26: 118-124.

[12] Steffens RF, Andrykowski MA (2016). Posttraumatic Growth Inventory: Overview. Comprehensive Guide to Post-Traumatic Stress Disorders. 2016: 2203-2219.

[13] Greup SR, Kaal SEJ, Jansen R, Manten-Horst E, Thong MSY, van der Graaf WTA, Prins JB, Husson O (2018). Post-Traumatic Growth and Resilience in Adolescent and Young Adult Cancer Patients: An Overview. J Adolesc Young Adult Oncol 7: 1-14.
[14] Lim JW (2019). The role of post-traumatic growth in promoting healthy behavior for couples coping with cancer. Support Care Cancer 27 (3): 829-838.

[15] Romeo A, Ghiggia A, Tesio V, Di Tella M, Torta R, Castelli L (2017). Post-traumatic growth, distress and attachment style among women with breast cancer. J Psychosoc Oncol 35 (3): 309-322.

[16] Ozcetin YSU, Hicdurmaz D (2017). Relations of post-traumatic growth and resilience in cancer experience. Eur Psychiatry 2017: S 672.

[17] Ruini C, Albieri E, Vescovelli F (2016). Post-Traumatic Growth, Psychological Well-Being, and Distress. Comprehensive Guide to Post-Traumatic Stress Disorder 2016: 1731-1754.

[18] Peng X, Su Y, Huang W, Hu X (2019). Status and factors related to posttraumatic growth in patients with lung cancer: A STROBE-compliant article. Medicine (Baltimore) 98: e14314.

[19] Dexue Z, Guoyun J, Hui H (2017). Effect of mental elasticity on self-perceived burden of lung cancer patients in chemotherapy. International Journal of Pathology and Clinical Medicine 37: 1025-1032.

[20] Kuijer RG, Buunk BP, De Jong GM, Ybema JF, Sanderman R (2004). Effects of a brief intervention program for patients with cancer and their partners on feelings of inequity, relationship quality and psychological distress. Psychooncology 13 (5): 321-334.

[21] Thomadaki, Olga O (2017). Bereavement, post-traumatic stress and post-traumatic growth: through the lenses of positive psychology. Eur J Psychotraumatol 8 (sup 4): 1351220.

[22] Seiler A, Jenewein J (2019). Resilience in Cancer Patients. Front Psychiatry 10: 208.

[23] Li L, Wang Q, Wang X, Jia XJ (2017). A study on the relationship between illness perception and post-traumatic growth in patients with breast cancer after surgery. Journal of Nurses Training 32 (14): 1312-1315.

[24] Wu ML (2010). Structural equation model: the operation and application of Amos. Second edition. Chongqing: Chongqing University Press (chapter 1, 2).

[25] Mei YQ, Li HuipingP, Yang YJ, Su D, Ma L, ZHang T, Dou Wj (2015). Reliability and Validity of Chinese Version of the Brief Illness Perception Questionnaire in Patients with Breast Cance. Journal of Nursing 22 (24): 11-14.

[26] Ye ZJ, Liu ML, Luo DM, Zeng Z, Hu GY, Zhu Yun F, Qiu H Z (2016). Reliability and validity test of 10 item Connor Davidson Resilience Scale in parents' group of children with cancer. Chinese Nursing research 30 (33): 4118-4122.

[27] Liu J, Feng XW, Liu YP (2015). Posttraumatic growth level and influencing factors analysis for patients with lung cancer. Chinese Journal of Modern Nursing 21 (25): 3029-3032, 3033.

[28] Ren YY (2012). The Development and preliminary clinical application of self-perceived burden scale for cancer patients. Dissertation, Shandong First Medical University.

[29] Akazawa T, Akechi T, Morita T, Miyashita M, Sato K, Tsuneto S, Shima Y, Furukawa TA (2010). Self-perceived burden in terminally ill cancer patients: a categorization of care strategies based on bereaved family members' perspectives. J Pain Symptom Manage 40: 224-234. 
[30] Song Q, Ji Q, Xiao J, Li F, Wang L, Chen Y, Xu Y, Jiao S (2018) miR-409 Inhibits Human Non-Small-Cell Lung Cancer Progression by Directly Targeting SPIN 1. Mol Ther Nucleic Acids 13: 154-163.

[31] Choi S, Ryu E (2018). Effects of symptom clusters and depression on the quality of life in patients with advanced lung cancer. Eur J Cancer Care (Engl) 27: 1-8.

[32] von Verschuer U, Schnell R, Tessen HW, Eggert J, Binninger A, Spring L, Jänicke M, Marschner N, Collective Name: TLK-Group (2017). Treatment, outcome and quality of life of 1239 patients with advanced non-small cell lung cancer - final results from the prospective German TLK cohort study. Lung Cancer 112: 216-224.

[33] Chabowski M, Polański J, Jankowska-Polańska B, Janczak D, Rosińczuk J (2018). Is nutritional status associated with the level of anxiety, depression and pain in patients with lung cancer. J Thorac Dis 10: 2303-2310.

[34] Sparla A, Flach-Vorgang S, Villalobos M, Krug K, Kamradt M, Coulibaly K, Szecsenyi J, Thomas M, Gusset-Bährer S, Ose D (2017). Reflection of illness and strategies for handling advanced lung cancer - a qualitative analysis in patients and their relatives. BMC Health Serv Res 17: 173.

[35] Gu W, Xu YM, Zhong BL (2018). Health-related quality of life in Chinese inpatients with lung cancer treatedin large general hospitals: across-sectional study. BMJ Open 8: e019873.

[36] Kuijer RG, Buunk BP, Ybema JF, Wobbes T (2002). The relation between perceived inequity, marital satisfaction and emotions among couples facing cancer. Br J Soc Psychol 41: 39-56.

[37] McPherson CJ, Wilson KG, Chyurlia L, Leclerc C (2010). The balance of give and take in caregiver-partner relationships: An examination of self-perceived burden, relationship equity, and quality of life from the perspective of care recipients following stroke. Rehabil Psychol 55: 194-203.

[38] Chen JE, Lou VW, Jian H, Zhou Z, Yan M, Zhu J, Li G, He Y (2018). Objective and subjective financial burden and its associations with health-related quality of life among lung cancer patients. Support Care Cancer 26: 1265-1272.
[39] Huang HY, Shi JF, Guo LW, et al (2017). Expenditure and financial burden for the diagnosis and treatment of colorectal cancer in China: a hospital-based, multicenter, cross-sectional survey. Chin J Cancer 36: 41.

[40] Romeo A, Di Tella M, Ghiggia A, Tesio V, Gasparetto E, Stanizzo MR, Torta R, Castelli L (2019). The Traumatic Experience of Breast Cancer: Which Factors Can Relate to the Post-traumatic Outcomes. Front Psychol 10: 891.

[41] Broadbent E, Wilkes C, Koschwanez H, Weinman J, Norton S, Petrie KJ (2015). A systematic review and meta-analysis of the Brief Illness Perception Questionnaire. Psychol Health 30: 1361-1385.

[42] Silva SM, Moreira HC, Canavarro MC (2012). Examining the links between perceived impact of breast cancer and psychosocial adjustment: the buffering role of posttraumatic growth. Psychooncology 21: 409-418.

[43] Dale SK, Cohen MH, Kelso GA, Cruise RC, Weber KM, Watson C, Burke-Miller JK, Brody LR (2014). Resilience among women with HIV: Impact of silencing the self and socioeconomic factors. Sex Roles 70: 221-231.

[44] Zhai J, Huang Y, Gao X, Jiang H, Xu J (2014). Post-trauma growth in a mainland Chinese population with chronic skin disease. Int J Dermatol 53: 450-457.

[45] Matzka M, Mayer H, Köck-Hódi S, Moses-Passini C, Dubey C, Jahn P, Schneeweiss S, Eicher M (2016). Relationship between Resilience, Psychological Distress and Physical Activity in Cancer Patients: A Cross-Sectional Observation Study. PLoS One 11: e0154496.

[46] Cameron LD, Muller C (2009). Psychosocial aspects of genetic testing. Curr Opin Psychiatry 22: 218-223.

[47] Hordijk PM, Broekhuizen BD, Butler CC, Coenen S, Godycki-Cwirko M, Goossens H, Hood K, Smith R, van Vugt SF, Little P, Verheij TJ, CollectiveName: GRACE Project Group (2015). Illness perception and related behaviour in lower respiratory tract infections - a European study. Fam Pract 32: 152-158. 